Modern Physics Letters B, to be published as "Brief Review" - cond-mat/9403033

(C) World Scientific Publishing Company

\title{
EXACTLY SOLVABLE SCALING THEORY OF CONDUCTION IN DISORDERED WIRES
}

\author{
C. W. J. Beenakker \\ Instituut-Lorentz, University of Leiden \\ P.O. Box 9506, 2300 RA Leiden, The Netherlands
}

Submitted March 1994

\section{ABSTRACT}

Recent developments are reviewed in the scaling theory of phase-coherent conduction through a disordered wire. The Dorokhov-Mello-Pereyra-Kumar equation for the distribution of transmission eigenvalues has been solved exactly, in the absence of timereversal symmetry. Comparison with the previous prediction of random-matrix theory shows that this prediction was highly accurate - but not exact: The repulsion of the smallest eigenvalues was overestimated by a factor of two. This factor of two resolves several disturbing discrepancies between random-matrix theory and microscopic calculations, notably in the magnitude of the universal conductance fluctuations in the metallic regime, and in the width of the log-normal conductance distribution in the insulating regime.

\section{Introduction}

In 1980, Anderson, Thouless, Abrahams, and Fisherl proposed a "new method for a scaling theory of localization", based on Landauer's interpretation of electrical conduction as quantum mechanical transmission. 2 They considered a one-dimensional (1D) chain with weak scattering (mean free path $l$ much greater than the Fermi wave length $\lambda_{\mathrm{F}}$ ), and computed how the transmission probability $T$ scales with the chain length $L$. For $L>l$ an exponential decay was obtained, demonstrating localization. In the following decade the scaling theory of $1 \mathrm{D}$ localization was developed in great detail, ${ }^{3-7}$ and the complete distribution $P(T, L)$ of the transmission probability was found (and hence of the conductance $G=T \times 2 e^{2} / h$ ). One can thus regard the problem of $1 \mathrm{D}$ localization as solved, at least for the case of weak scattering.

A real metal wire is not one-dimensional. Typically, the width $W$ is much greater than $\lambda_{\mathrm{F}}$, so that the number $N$ of transverse modes at the Fermi level is much greater than one. Instead of a single transmission probability $T$, one now has $N$ transmission eigenvalues $T_{1}, T_{2}, \ldots T_{N}$. (The numbers $T_{n} \in[0,1]$ are the eigenvalues of the matrix product $t t^{\dagger}$, where $t$ is the $N \times N$ transmission matrix of the wire.) To obtain the distribution of the conductance

$$
G=\frac{2 e^{2}}{h} \sum_{n=1}^{N} T_{n},
$$


one now needs the joint probability distribution $P\left(T_{1}, T_{2}, \ldots T_{N}, L\right)$. This distribution differs essentially from the distribution in the $1 \mathrm{D}$ chain, because of strong correlations between the transmission eigenvalues. These correlations originate from a "repulsion" of nearby eigenvalues. As a consequence of this eigenvalue repulsion, the localization length is increased by a factor of $N$ in comparison to the $1 \mathrm{D}$ case. One can therefore distinguish a metallic and an insulating regime. On length scales $l<L<N l$ the conductance decreases linearly rather than exponentially with $L$. This is the (diffusive) metallic regime, where mesoscopic effects as weak localization and universal conductance fluctuations (UCF) occur. The insulating regime of exponentially small conductance is entered for wire lengths $L>N l$.

A scaling theory of localization in multi-mode wires was pioneered by Dorokhov, 目 and independently by Mello, Pereyra, and Kumar. 9 The DMPK scaling equation,

$$
\begin{aligned}
l \frac{\partial P}{\partial L} & =\frac{2}{\beta N+2-\beta} \sum_{i=1}^{N} \frac{\partial}{\partial \lambda_{i}} \lambda_{i}\left(1+\lambda_{i}\right) J \frac{\partial}{\partial \lambda_{i}} J^{-1} P, \\
J & =\prod_{i<j}\left|\lambda_{j}-\lambda_{i}\right|^{\beta},
\end{aligned}
$$

describes the evolution of the distribution function $P\left(\lambda_{1}, \lambda_{2}, \ldots \lambda_{N}, L\right)$ in an ensemble of disordered wires of increasing length. The variables $\lambda_{n} \in[0, \infty)$ are simply related to the transmission eigenvalues by $\lambda_{n} \equiv\left(1-T_{n}\right) / T_{n}$. The ensemble is characterized by a mean free path $l$ and by a symmetry index $\beta$, which takes on the values 1,2 , and 4 depending on the presence or absence of time-reversal and spin-rotation symmetry ( $\beta=2$ in the presence of a magnetic field; otherwise, $\beta=1$ or 4 in the absence or presence of spin-orbit scattering).

The DMPK equation has the form of a diffusion equation in eigenvalue space. The function $J$ which couples the degrees of freedom is the Jacobian from the space of scattering matrices to the space of transmission eigenvalues. The similarity to diffusion in real space has been given further substance by the demonstration $\mathbb{E}$ that Eq. (2) holds on length scales $\gg l$ regardless of the microscopic scattering properties of the conductor (one-parameter scaling). $\mathrm{Eg}$ (2) was derived by Dorokhov, (for $\beta=2$ ) and by Mello, Pereyra, and Kumar, (for $\beta=1$, with generalizations to $\beta=2,4$ in Refs. 11,12 ) by computing the incremental change of the transmission eigenvalues upon attachment of a thin slice to the wire. It is assumed that the conductor is weakly disordered $\left(l \gg \lambda_{\mathrm{F}}\right)$, so that the scattering in the thin slice can be treated by perturbation theory. A key simplification is the isotropy assumption that the flux incident in one scattering channel is, on average, equally distributed among all outgoing channels. This assumption restricts the applicability of the DMPK equation to a wire geometry $(L \gg W)$, since it ignores the finite time scale for transverse diffusion.

The DMPK equation has been studied extensively for more than ten years. The strong coupling of the scattering channels by the Jacobian prevented an exact solution by standard methods. The problem simplifies drastically deep in the localized regime $(L \gg N l)$, when the scattering channels become effectively decoupled. 
Pichard 13 has computed from Eq. (2) the log-normal distribution of the conductance in this regime, and has found an excellent agreement with numerical simulations. In the metallic regime $(L \ll N l)$, Mello and Stone11. 14 were able to compute the first two moments of the conductance, in precise agreement with the diagrammatic perturbation theory of weak localization and UCF. Moregeneral calculations of the weak localization effect 15 and of universal fluctuations 16 [for arbitrary transport properties of the form $A=\sum_{n} f\left(T_{n}\right)$ ] have been developed, based on linearization of Eq. (2) in the fluctuations of the $\lambda$ 's around their mean positions (valid in the large- $N$ metallic regime, when the fluctuations are small). None of these approaches was capable of finding the full distribution function. The purpose of this paper is to review some recent work by B. Rejaei and the author, 17 in which the DMPK scaling equation has been solved exactly for the case $\beta=2$.

\section{Random-matrix theory and the $1 / 8-2 / 15$ puzzle}

There existed a special and urgent reason for wanting the full distribution function of the transmission eigenvalues. We are referring to a disturbing discrepancy 18 between the random-matrix theory of UCF and the established diagrammatic perturbation theory. In order to appreciate the significance of the recent developments, it seems worthwhile to discuss this issue in some "historical" perspective.

In the sixties, Wigner, Dyson, Mehta, and others developed random-matrix theory (RMT) into a powerful tool to study the statistics of energy levels measured in nuclear reactions. 19 It was shown that the fluctuations in the energy level density are governed by level repulsion. Mathematically, level repulsion originates from the Jacobian $J=\prod_{i<j}\left|E_{j}-E_{i}\right|^{\beta}$ of the transformation from matrix space to eigenvalue space, which depends on the symmetry of the Hamiltonian ensemble but is independent of the mean level density.20 This universality is at the origin of the remarkable success of RMT in nuclear physics.21 The universality of the level fluctuations is expressed by the celebrated Dyson-Mehta formula 22 for the variance of a linear statistic $A=\sum_{n} a\left(E_{n}\right)$ on the energy levels $E_{n}$. (The quantity $A$ is called a linear statistic because products of different $E_{n}$ 's do not appear, but the function $a(E)$ may well depend non-linearly on $E$.) The Dyson-Mehta formula reads

$$
\operatorname{Var} A=\frac{1}{\beta} \frac{1}{\pi^{2}} \int_{0}^{\infty} d k|a(k)|^{2} k,
$$

where $a(k)=\int_{-\infty}^{\infty} d E \mathrm{e}^{\mathrm{i} k E} a(E)$ is the Fourier transform of $a(E)$. Eq. (4) shows that: 1 . The variance is independent of microscopic parameters; 2 . The variance has a universal $1 / \beta$-dependence on the symmetry index.

In a seminal 1986-paper,23 Imry proposed to apply RMT to the phenomenon of universal conductance fluctuations, which was discovered using diagrammatic perturbation theory by Al'tshulen 24 and Lee and Stone.25 UCF is the occurrence of sample-to-sample fluctuations in the conductance which are of order $e^{2} / h$ at zero temperature, independent of the size of the sample or the degree of disorder as long as the conductor remains in the diffusive metallic regime. The relationship 
between the statistics of energy levels measured in nuclear reactions on the one hand, and the statistics of conductance fluctuations measured in transport experiments on the other hand, was used by Muttalib, Pichard, and Stone 26 to develop a randommatrix theory of quantum transport. (For a review, see Ref. 27.) The RMT of quantum transport differs from the RMT of level statistics in two essential ways.

(i) The first is that the transmission eigenvalues $T_{n}$ are not the eigenvalues of the scattering matrix. Instead they are the eigenvalues of $t t^{\dagger}$, where the transmission matrix $t$ is an $N \times N$ submatrix of the $2 N \times 2 N$ scattering matrix of the conductor. It turns out that the repulsion of the variables $\lambda_{n} \equiv\left(1-T_{n}\right) / T_{n}$ takes the same form as the repulsion of the energy levels $E_{n}$. More precisely, the Jacobian (3) in terms of the $\lambda$ 's has the same form as for level statistics. Random-matrix theory is based on the fundamental assumption that all correlations between the eigenvalues are due to the Jacobian. If all correlations are due to the Jacobian, then the probability distribution $P\left(\lambda_{1}, \lambda_{2}, \ldots \lambda_{N}\right)$ of the $\lambda$ 's should have the form $P \propto J \prod_{i} p\left(\lambda_{i}\right)$, or equivalently,

$$
\begin{aligned}
& P\left(\left\{\lambda_{n}\right\}\right)=C \exp \left[-\beta\left(\sum_{i<j} u\left(\lambda_{i}, \lambda_{j}\right)+\sum_{i} V\left(\lambda_{i}\right)\right)\right], \\
& u\left(\lambda_{i}, \lambda_{j}\right)=-\ln \left|\lambda_{j}-\lambda_{i}\right|,
\end{aligned}
$$

with $V=-\beta^{-1} \ln p$ and $C$ a normalization constant. Eq. (5) has the form of a Gibbs distribution at temperature $\beta^{-1}$ for a fictitious system of classical particles on a line in an external potential $V$, with a logarithmically repulsive interaction $u$. All microscopic parameters are contained in the single function $V(\lambda)$. The logarithmic repulsion is independent of microscopic parameters, because of its geometric origin.

(ii) The second difference is that the correlation function of the $\lambda$ 's is not translationally invariant, due to the positivity constraint on $\lambda$. This constraint $\lambda \geq 0$ follows directly from unitarity of the scattering matrix. In contrast, the correlation function in the RMT of energy levels is translationally invariant over the energy range of interest. Because of this complication, it could not be shown that the universality of the fluctuations is generic for arbitrary linear statistics on the transmission eigenvalues. In particular, no formula with the generality of the Dyson-Mehta formula (4) could be derived. The lack of such a general theory was being felt especially since mesoscopic fluctuations in transport properties other than the conductance (both in conductors and superconductors) became of interest. Examples are the critical-current fluctuations in Josephson junctions, 28 conductance fluctuations at normal-superconductor interfaces, 29 and fluctuations in the shot-noise power of metals. 30 This obstacle towards the establishment of universality in the RMT of quantum transport was finally overcome in 1993, 18 when a technique was developed to compute correlation functions by a method of functional derivatives, which does not require translational invariance. The analogue could be obtained of the DysonMehta formula for the variance of a linear statistic $A=\sum_{n} f\left(T_{n}\right)$ on the 
transmission eigenvalues:

$$
\operatorname{Var} A=\frac{1}{\beta} \frac{1}{\pi^{2}} \int_{0}^{\infty} d k|F(k)|^{2} k \tanh (\pi k) .
$$

The function $F(k)$ is defined in terms of the function $f(T)$ by the transform

$$
F(k)=\int_{-\infty}^{\infty} d x \mathrm{e}^{\mathrm{i} k x} f\left(\frac{1}{1+\mathrm{e}^{x}}\right) .
$$

The formula (7) demonstrates that the universality which was the hallmark of UCF is generic for a whole class of transport properties, viz. those which are linear statistics on the transmission eigenvalues.

The probability distribution (5) was justified by a maximum-entropy principle for quasi-1D conductors.26 Quasi-1D means $L \gg W \gg \lambda_{\mathrm{F}}$. In this limit one can assume that the distribution of scattering matrices is only a function of the transmission eigenvalues (isotropy assumption). The distribution (5) then maximizes the information entropy subject to the constraint of a given density of eigenvalues. The function $V(\lambda)$ is determined by this constraint and is not specified by RMT.

It was initially believed that Eq. (5) would provide an exact description in the quasi-1D limit, if only $V(\lambda)$ were suitably chosen.27 However, the generalized DysonMehta formula (\$) demonstrates that RMT is not exact, not even in the quasi$1 \mathrm{D}$ limit. If one computes from Eq. (17) the variance of the conductance (1) [by substituting $f(T)=G_{0} T$, with $\left.G_{0}=2 e^{2} / h\right]$, one finds

$$
\operatorname{Var} G / G_{0}=\frac{1}{8} \beta^{-1}
$$

independent of the form of $V(\lambda)$. The diagrammatic perturbation theory 2425 of UCF gives instead

$$
\operatorname{Var} G / G_{0}=\frac{2}{15} \beta^{-1}
$$

for a quasi-1D conductor. The difference between the coefficients $\frac{1}{8}$ and $\frac{2}{15}$ is tiny, but it has the fundamental implication that the interaction between the $\lambda$ 's is not precisely logarithmic, or in other words, that there exist correlations between the transmission eigenvalues over and above those induced by the Jacobian.

The $\frac{1}{8}-\frac{2}{15}$ discrepancy raised the question what the true eigenvalue interaction would be in quasi-1D conductors. Is there perhaps a cutoff for large separation of the $\lambda$ 's? Or is the true interaction a many-body interaction, which can not be reduced to the sum of pairwise interactions? This transport problem has a counterpart in a closed system. The RMT of the statistics of the eigenvalues of a random Hamiltonian yields a probability distribution of the form (5) with a logarithmic repulsion between the energy levels.20 It was shown by Efetov 13 and by Al'tshuler and Shklovskil 32 that the logarithmic repulsion in a small disordered particle (length $L$, diffusion constant $D$ ) holds for energy separations small compared to the Thouless energy $E_{\mathrm{c}} \equiv \hbar D / L^{2}$. For larger separations the interaction potential decays 
algebraically. 33 As we shall discuss, the way in which the RMT of quantum transport breaks down is quite different.

\section{Non-logarithmic eigenvalue repulsion}

The method of solution of the DMPK equation is a mapping onto a model of non-interacting fermions, inspired by Sutherland's mapping of a different diffusion equation.34 The case $\beta=2$ is special, because for other values of $\beta$ the mapping introduces interactions between the fermions. The free-fermion problem has the character of a one-dimensional scattering problem in imaginary time, which can be solved exactly without great difficulties. The reader who is interested in "how it is done" is referred to Ref. 17. In this Brief Review we limit ourselves to presenting the solution and discussing its implications.

The DMPK equation (2) [with $\beta=2$ ] can be solved for arbitrary initial conditions. We consider the ballistic initial condition $\lim _{L \rightarrow 0} P=\prod_{i} \delta\left(\lambda_{i}\right)$, appropriate for the case of ideal contacts. The solution is given by the square root of the Jacobian (3) times the determinant of an $N$-dimensional matrix $M$. The determinant is the Slater determinant of the free-fermion problem. The square root of the Jacobian comes from the mapping of the DMPK equation onto the Schrödinger equation. The solution is

$$
\begin{aligned}
& P\left(\left\{\lambda_{n}\right\}, L\right)=C(L) J^{1 / 2}|\operatorname{Det} M| \\
& M_{n m}=\int_{0}^{\infty} d k \exp \left(-\frac{1}{4} k^{2} L / N l\right) \tanh \left(\frac{1}{2} \pi k\right) k^{2 m-1} \mathrm{P}_{\frac{1}{2}(\mathrm{i} k-1)}\left(1+2 \lambda_{n}\right),
\end{aligned}
$$

where $C(L)$ is a $\lambda$-independent normalization factor. Using an integral representation for the Legendre functions $\mathrm{P}_{\nu}$, the matrix elements (12) can be rewritten in terms of Hermite polynomials $\mathrm{H}_{2 m-1}$,

$$
M_{n m}=c \int_{\operatorname{arccosh}\left(1+2 \lambda_{n}\right)}^{\infty} d u \exp \left(-\frac{1}{4} u^{2} N l / L\right)\left(\cosh u-1-2 \lambda_{n}\right)^{-1 / 2} \mathrm{H}_{2 m-1}\left(\frac{1}{2} u \sqrt{N l / L}\right),
$$

where $c$ is another constant which can be absorbed in $C(L)$.

For $N=1$, the Jacobian $J \equiv 1$ and $\operatorname{Det} M=M_{11}$, so that Eq. (11) reduces to

$$
P(\lambda, L)=C(L) \int_{\operatorname{arccosh}(1+2 \lambda)}^{\infty} d u \exp \left(-\frac{1}{4} u^{2} l / L\right)(\cosh u-1-2 \lambda)^{-1 / 2} u .
$$

Normalization gives $C(L)=(2 \pi)^{-1 / 2}(l / L)^{3 / 2} \exp \left(-\frac{1}{4} L / l\right)$. This is Abrikosov's solution $\theta$ of the scaling equation for a $1 \mathrm{D}$ chain. ${ }^{a}$ This solution is $\beta$-independent

\footnotetext{
${ }^{a}$ This solution (14) the 1D scaling equation was actually obtained as early as 1959 by Gertsenshtein and Vasil'ev 35 in a paper entitled "Waveguides with random inhomogeneities and Brownian motion in the Lobachevsky plane." This remarkable paper on the exponential decay of radio-waves due to weak disorder contains many of the results which were rederived in the eighties for the problem of $1 \mathrm{D}$ localization of electrons. ${ }^{3-7}$ The paper was noticed in the optical literature, 36 but apparently not among solid-state physicists.
} 
( $\beta$ drops out of Eq. (2) for $N=1$ ). Equation (11) generalizes the 1D-chain solution to arbitrary $N$, for the case $\beta=2$.

The Slater determinant can be evaluated in closed form in the metallic regime $L \ll N l$ and in the insulating regime $L \gg N l$. In both regimes the probability distribution takes the form (5) of a Gibbs distribution with a parameter-independent two-body interaction $u\left(\lambda_{i}, \lambda_{j}\right)$, as predicted by RMT. However, the interaction differs from the logarithmic repulsion (6) of RMT. Instead, it is given by 17

$$
u\left(\lambda_{i}, \lambda_{j}\right)=-\frac{1}{2} \ln \left|\lambda_{j}-\lambda_{i}\right|-\frac{1}{2} \ln \left|\operatorname{arcsinh}^{2} \lambda_{j}^{1 / 2}-\operatorname{arcsinh}^{2} \lambda_{i}^{1 / 2}\right| .
$$

The eigenvalue interaction (15) is different for weakly and for strongly transmitting scattering channels: $u \rightarrow-\ln \left|\lambda_{j}-\lambda_{i}\right|$ for $\lambda_{i}, \lambda_{j} \ll 1$, but $u \rightarrow-\frac{1}{2} \ln \left|\lambda_{j}-\lambda_{i}\right|$ for $\lambda_{i}, \lambda_{j} \gg 1$. For weakly transmitting channels it is twice as small as predicted by considerations based solely on the Jacobian, which turn out to apply only to the strongly transmitting channels.

In the metallic regime $L \ll N l$, the method of functional derivatives of Ref. 18 can still be used to compute the variance of a linear statistic, since this method works for any two-body interaction. Instead of Eq. (7), one now obtains for the variance the formula

$$
\begin{aligned}
& \operatorname{Var} A=\frac{1}{\beta} \frac{1}{\pi^{2}} \int_{0}^{\infty} d k \frac{k|F(k)|^{2}}{1+\operatorname{cotanh}\left(\frac{1}{2} \pi k\right)}, \\
& F(k)=\int_{-\infty}^{\infty} d x \mathrm{e}^{\mathrm{i} k x} f\left(\frac{1}{\cosh ^{2} x}\right) .
\end{aligned}
$$

This result was obtained for $\beta=2$ from the exact solution given above,17 and independently for all $\beta \in\{1,2,4\}$ by the perturbative method of Chalker and Macêdo.16 Substitution of $f(T)=T$ now yields $\frac{2}{15}$ instead of $\frac{1}{8}$ for the coefficient of the UCF, thus resolving the discrepancy between Eqs. (9) and (10). The conclusion is that the discrepancy with RMT originated from a reduced repulsion of weakly transmitting channels.

In the insulating regime $L \gg N l$, all $\lambda$ 's are exponentially large, and the interaction (15) may be effectively simplified by $u\left(\lambda_{i}, \lambda_{j}\right)=-\frac{1}{2} \ln \left|\lambda_{j}-\lambda_{i}\right|$. This is a factor of two smaller than the interaction (6) predicted by RMT. This explains the factor-of-two discrepancy between the results of RMT and of numerical simulations for the width of the log-normal distribution of the conductance 13 RMT predicts Var $\ln G / G_{0}=-\left\langle\ln G / G_{0}\right\rangle$, which is twice as small as the result

$$
\text { Var } \ln G / G_{0}=-2\left\langle\ln G / G_{0}\right\rangle
$$

which follows from the exact solution of the DMPK equation for $\beta=2$. As shown by Pichard 13 the relationship (18) between mean and variance of $\ln G / G_{0}$ remains valid for other values of $\beta$, since both the mean and the variance have a $1 / \beta$ dependence on the symmetry index. 


\section{Outlook}

We conclude by mentioning some directions for future research. So far only the case $\beta=2$ of broken time-reversal symmetry has been solved exactly. 17 In that case the DMPK equation (2) can be mapped onto a free-fermion problem. For $\beta=1,4$ the Sutherland-type mapping is onto an interacting Schrödinger equation. It might be possible to solve this equation exactly too, using techniques developed recently for the Sutherland Hamiltonian. 37 From the work of Chalker and Macêdo 16 we know that the two-point correlation function of the eigenvalues in the large- $N$ limit has a simple $1 / \beta$-dependence on the symmetry index. This poses strong restrictions on a possible $\beta$-dependence of the eigenvalue interaction, which can only differ from the form (15) derived for $\beta=2$ on intervals $\Delta \lambda \simeq L / N l \ll 1$ comparable to the spacing between the $\lambda$ 's.

It might be possible to come up with another maximum-entropy principle, different from that of Muttalib, Pichard, and Stone, 26 which yields the correct eigenvalue interaction (15) instead of the logarithmic interaction (6). Slevin and Nagao 38 have proposed an alternative maximum-entropy principle, but their distribution function does not improve the agreement with Eq. (10). It would be particularly worthwhile to find an intuitive explanation for the halving of the logarithmic interaction for weakly transmitted scattering channels.

To go beyond quasi-one-dimensional geometries (long and narrow wires) remains an outstanding problem. A numerical study of Slevin, Pichard, and Muttalib 39 has indicated a significant break-down of the logarithmic repulsion for two- and threedimensional geometries (squares and cubes). A generalization of the DMPK equation (2) to higher dimensions has been the subject of some recent investigations. 40.4

It remains to be seen whether the method reviewed here for Eq. (2) is of use for that problem.

\section{Acknowledgements}

The research reviewed in this paper was carried out in collaboration with B. Rejaei. It was supported financially by the "Nederlandse organisatie voor Wetenschappelijk Onderzoek" (NWO) and by the "Stichting voor Fundamenteel Onderzoek der Materie" (FOM).

\section{References}

1. P. W. Anderson, D. J. Thouless, E. Abrahams, and D. S. Fisher, Phys. Rev. B22, 3519 (1980).

2. R. Landauer, IBM J. Res. Dev. 1, 223 (1957); Phil. Mag. 21, 863 (1970).

3. V. I. Mel'nikov, Fiz. Tverd. Tela 23, 782 (1981) [Sov. Phys. Solid State 23, 444 (1981)].

4. A. A. Abrikosov, Solid State Comm. 37, 997 (1981).

5. P. D. Kirkman and J. B. Pendry, J. Phys. C17, 5707 (1984).

6. N. Kumar, Phys. Rev. B31, 5513 (1985).

7. P. A. Mello, J. Math. Phys. 27, 2876 (1986). 
8. O. N. Dorokhov, Pis'ma Zh. Eksp. Teor. Fiz. 36, 259 (1982) [JETP Lett. 36, 318 (1982)].

9. P. A. Mello, P. Pereyra, and N. Kumar, Ann. Phys. 181, 290 (1988).

10. P. A. Mello and B. Shapiro, Phys. Rev. B37, 5860 (1988).

11. P. A. Mello and A. D. Stone, Phys. Rev. B44, 3559 (1991).

12. A. M. S. Macêdo and J. T. Chalker, Phys. Rev. B46, 14985 (1992).

13. J.-L. Pichard, in: Quantum Coherence in Mesoscopic Systems, ed. by B. Kramer, NATO ASI Series B254 (Plenum, New York, 1991).

14. P. A. Mello, Phys. Rev. Lett. 60, 1089 (1988).

15. C. W. J. Beenakker, Phys. Rev. B49, 2205 (1994).

16. J. T. Chalker and A. M. S. Macêdo, Phys. Rev. Lett. 71, 3693 (1993).

17. C. W. J. Beenakker and B. Rejaei, Phys. Rev. Lett. 71, 3689 (1993); Phys. Rev. B (March 15, 1994).

18. C. W. J. Beenakker, Phys. Rev. Lett. 70, 1155 (1993); Phys. Rev. B47, 15763 (1993).

19. C. E. Porter, ed., Statistical Theories of Spectra: Fluctuations (Academic, New York, 1965).

20. M. L. Mehta, Random Matrices (Academic, New York, 1991).

21. T. A. Brody, J. Flores, J. B. French, P. A. Mello, A. Pandey, and S. S. M. Wong, Rev. Mod. Phys. 53, 385 (1981).

22. F. J. Dyson and M. L. Mehta, J. Math. Phys. 4, 701 (1963).

23. Y. Imry, Europhys. Lett. 1, 249 (1986).

24. B. L. Al'tshuler, Pis'ma Zh. Eksp. Teor. Fiz. 41, 530 (1985) [JETP Lett. 41, 648, (1985)].

25. P. A. Lee and A. D. Stone, Phys. Rev. Lett. 55, 1622 (1985).

26. K. A. Muttalib, J.-L. Pichard, and A. D. Stone, Phys. Rev. Lett. 59, 2475 (1987).

27. A. D. Stone, P. A. Mello, K. A. Muttalib, and J.-L. Pichard, in: Mesoscopic Phenomena in Solids, ed. by B. L. Al'tshuler, P. A. Lee, and R. A. Webb (North-Holland, Amsterdam, 1991).

28. C. W. J. Beenakker, Phys. Rev. Lett. 67, 3836 (1991); 68, 1442(E) (1992).

29. Y. Takane and H. Ebisawa, J. Phys. Soc. Japan 60, 3130 (1991).

30. M. J. M. de Jong and C. W. J. Beenakker, Phys. Rev. B46, 13400 (1992).

31. K. B. Efetov, Adv. Phys. 32, 53 (1983).

32. B. L. Al'tshuler and B. I. Shklovskiı̌, Zh. Eksp. Teor. Fiz. 91, 220 (1986) [Sov. Phys. JETP 64, 127 (1986)].

33. R. A. Jalabert, J.-L. Pichard, and C. W. J. Beenakker, Europhys. Lett. 24, 1 (1993).

34. B. Sutherland, Phys. Rev. A5, 1372 (1972).

35. M. E. Gertsenshtein and V. B. Vasil'ev, Teor. Veroyatn. Primen. 4, 424 (1959) [Theor. Probab. Appl. 4, 391 (1959)].

36. G. C. Papanicolaou, SIAM J. Appl. Math. 21, 13 (1971).

37. B. D. Simons, P. A. Lee, and B. L. Al'tshuler, Phys. Rev. Lett. 70, 4122 (1993).

38. K. Slevin and T. Nagao, Phys. Rev. Lett. 70, 635 (1993); and preprint.

39. K. Slevin, J.-L. Pichard, and K. A. Muttalib, J. Physique I (France) 3, 1387 (1993).

40. P. A. Mello and S. Tomsovic, Phys. Rev. Lett. 67, 342 (1991).

41. J. T. Chalker and M. Bernhardt, Phys. Rev. Lett. 70, 982 (1993). 